\title{
The Selective Antibacterial Activity of the Mixed Systems Containing Myristic Acid against Staphylococci
}

\author{
Minako Okukawa, Yuika Yoshizaki, Shigekazu Yano, and Yoshimune Nonomura* \\ Department of Biochemical Engineering, Graduate School of Science and Engineering, Yamagata University, 4-3-16 Jonan, Yonezawa 992-8510, \\ JAPAN
}

\begin{abstract}
Fatty acids and their derivatives are interesting cosmetic ingredients because they show the selective antibacterial activity against Staphylococcus aureus (S. aureus). However, the antibacterial activity in mixed systems containing several active ingredients is unclear because previous studies focused antibacterial systems containing one kind of fatty acid. In the present study, the minimal inhibitory concentration (MIC) and the fractional inhibitory concentration (FIC) were evaluated for myristic acid/ lauric acid, myristic acid/palmitoleic acid, and myristic acid/lactic acid mixed systems to show the effect of the coexisting components on the selective antibacterial activity of myristic acid. In the myristic acid/ palmitoleic acid mixed system, the antibacterial activity against $S$. aureus was enhanced by additive effect, whereas the antibacterial activity was not observed against $S$. epidermidis. On the other hand, the myristic acid/lauric acid mixed system showed antibacterial activity against $S$. epidermidis: Lauric acid impaired the selectivity of antibacterial activity of myristic acid. These results suggest that the selective activity of myristic acid varies with the additives. The present findings are useful for designing formulations of cosmetics and body cleansers containing myristic acid.
\end{abstract}

Key words: selective antibacterial activity, combination effect, fatty acid, minimal inhibitory concentration, fractional inhibitory concentration

\section{Introduction}

Fatty acids are widely added in body cleaners and cosmetics because of their excellent foaming ability, cleansing ability, and low toxicity ${ }^{1,2)}$. In particular, some fatty acids have selective antibacterial properties; they show antibacterial activity against Staphylococcus aureus (S. aureus) which causes atopic dermatitis and skin roughness, while they do not against Staphylococcus epidermidis (S. epidermidis) which contributes to the immune system of human skin ${ }^{3-5)}$. Therefore, fatty acids have been focused as key materials for body cleaners and skin care cosmetics. In previous studies, we reported that the alkyl chain length, unsaturated degree, and metal ions of fatty acids affect their antibacterial activity against Staphylococci, and that several fatty acids such as myristic acid and palmitoleic acid and their derivatives selectively inhibit the growth of S. aureus ${ }^{6-10}$.

The antimicrobial activity of fatty acids in mixed system with other components is unclear, because only a single fatty acid was added in these evaluation systems. However, cosmetics and body cleansers contain organic acids such as citric acid and lactic acid, as well as several types of fatty acids, each of which has an antibacterial effect ${ }^{11-14)}$. Therefore, findings of antibacterial activity in mixed systems containing multiple antibacterial agents is important for the preparation of effective antibacterial systems. The checkerboard method is useful for evaluating the antibacterial activity of systems containing multiple ingredients. The synergistic or antagonistic effects of two antibacterial agents can be determined by calculating the fractional inhibitory concentration (FIC) index based on the growth inhibition effect of the combination of the two agents ${ }^{15,16)}$. Recently, we evaluated the antibacterial activity of two-component mixed systems containing 1,2-dodecanediol and additives against Staphylococci and reported that the addition of myristic acid inhibited the antibacterial activity of 1,2-dodecanediol against $S$. epidermidis ${ }^{17}$.

In the present study, we evaluated the antibacterial ac-

\footnotetext{
* Correspondence to: Yoshimune Nonomura, Department of Biochemical Engineering, Graduate School of Science and Engineering, Yamagata University, 4-3-16 Jonan, Yonezawa 992-8510, JAPAN

E-mail: nonoy@yz.yamagata-u.ac.jp

Accepted April 29, 2021 (received for review March 10, 2021)

Journal of Oleo Science ISSN 1345-8957 print / ISSN 1347-3352 online

http://www.jstage.jst.go.jp/browse/jos/ http://mc.manusriptcentral.com/jjocs




tivity of two-component mixed system containing myristic acid which shows the selective antibacterial activity and other antibacterial ingredients against $S$. aureus and $S$. epidermidis. The additives were lauric acid, which has the maximum antibacterial activity among saturated fatty acids, palmitoleic acid, which has characteristic selective antibacterial activity, and lactic acid, which is also used as a degradation preventing agent in foods and a topical skin inflammation treatment agent ${ }^{10,18,19)}$. The antibacterial activity of these substances was determined using minimal inhibitory concentration (MIC), which is the minimum drug concentration required to suppress bacterial growth, while the combined effect of the two-component mixture was evaluated based on the FIC index. The findings of the present study will be useful in designing skin care and cleansing products that focus on the human skin flora.

\section{Experimental}

\subsection{Materials}

The molecular structures of the materials evaluated in the present study are shown in Fig. 1. Myristic acid (C14:0 fatty acid, $\geq 99 \%$ ) was purchased from Sigma-Aldrich Co. LLC (St. Louis, USA). Lactic acid, lauric acid (C12:0 fatty acid, $\geq 99 \%$ ), palmitoleic acid (C16:1 fatty acid, $\geq 99 \%$ ) and $0.1 \mathrm{~mol} \mathrm{~L}^{-1}$ phosphate buffer solution $(\mathrm{pH} 6)$ were obtained from Fujifilm Wako Pure Chemical Industries Ltd. (Osaka, Japan). Ethanol, sodium chloride, beef extract, and polypeptone were purchased from Junsei Chemical Co. (Tokyo, Japan), Kanto Chemical Co., Inc. (Tokyo, Japan), Nacalai Tesque Inc. (Kyoto, Japan), and Nihon Pharmaceutical Co., Ltd. (Tokyo, Japan), respectively. Agar powder was purchased from Nissui Pharmaceutical Co., Ltd. (Tokyo, Japan). S. aureus (NBRC13276) and S. epidermidis (NBRC12993) were obtained from the National Institute of Technology and Evaluation (Tokyo, Japan). Water was purified using a Demi-Ace Model DX-15 demineralizer(Kurita Water Industries Ltd., Tokyo, Japan).

\subsection{Preparation of culture medium and bacterial disper- sion}

Before the cultivation of $S$. aureus and $S$. epidermidis, a medium containing beef extract $(0.15 \mathrm{~g})$, polypeptone (0.30 g), sodium chloride $(0.15 \mathrm{~g})$, and water $(30 \mathrm{~mL})$ was sterilized in an autoclave at $121^{\circ} \mathrm{C}$ for $20 \mathrm{~min}$. The cultures of $S$. aureus and $S$. epidermidis were prepared by shaking $30 \mathrm{~mL}$ of the medium containing one colony for 24 $\mathrm{h}$ at $37^{\circ} \mathrm{C}(145 \mathrm{rpm})$. For the MIC evaluation and the assays using the checkerboard method, the medium containing beef extract $(1.50 \mathrm{~g})$, polypeptone (3.00 g), sodium chloride $(1.50 \mathrm{~g})$, and pH 6 phosphate buffer $(150 \mathrm{~mL})$ were sterilized in an autoclave at $121^{\circ} \mathrm{C}$ for $20 \mathrm{~min}$.<smiles>CCCCCCCCCCCCCC(=O)O</smiles>

(c)

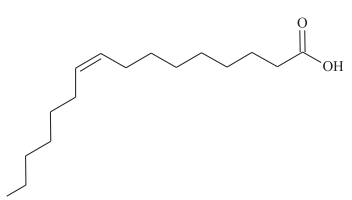<smiles>CCCCCCCCCCCC(=O)O</smiles>

(d)<smiles>CC(O)C(=O)O</smiles>

Fig. 1 Molecular structure of (a) Myristic acid, (b) Lauric acid, (c) Palmitoleic acid, and (d) Lactic acid.

Table 1 MIC values of myristic acid, lauric acid, palmitoleic acid and lactic acid. From references 10) and 17). In this study, MICs were re-evaluated under more precise conditions. As a result, we obtained more accurate values for the MIC for myristic acid, which were different from the data reported in 10).

\begin{tabular}{lcc}
\hline \multirow{2}{*}{ Substances } & \multicolumn{2}{c}{ MIC $/ \mu \mathrm{g} \mathrm{mL}^{-1}$} \\
\cline { 2 - 3 } & $\begin{array}{c}\text { S. aureus } \\
\text { NBRC13276 }\end{array}$ & $\begin{array}{c}\text { S. epidermidis } \\
\text { NBRC12993 }\end{array}$ \\
\hline Myristic acid & 157 & $>2500$ \\
Lauric acid & 18.8 & 18.8 \\
Palmitoleic acid & 18.8 & $>400$ \\
Lactic acid & 4000 & 3200 \\
\hline
\end{tabular}

\subsection{Evaluation of the combined effect of antibacterial} components based on checkerboard method

The effects of the addition of lauric acid, palmitoleic acid, and lactic acid on the antibacterial activity of myristic acid were evaluated using the checkerboard method, which was a typical evaluation method for mixed antibacterial systems. In the checkerboard method, a combined effect was determined based on the FIC index, which was calculated from the MIC of a mixture containing two types of ingredients. We have reported the MICs of myristic acid and three additives in a single component against $S$. aureus and $S$. epidermidis in previously (Table 1) ${ }^{10,17)}$.

Here, the antibacterial activity of the mixtures containing myristic acid and the additives in the concentrations of 1/64 to 4 times the MIC was evaluated: The concentration ranges of myristic acid and additives were as follows: 2.45 $\sim 628 \mu \mathrm{g} \mathrm{mL}^{-1}$ (myristic acid for $S$. aureus), 6.25 1600 $\mu \mathrm{g} \mathrm{mL}{ }^{-1}$ (myristic acid for S. epidermidis), $0.29 \sim 75.2 \mu \mathrm{g}$ $\mathrm{mL}^{-1}$ (lauric acid for $S$. aureus, $S$. epidermidis), 0.29〜 $75.2 \mu \mathrm{g} \mathrm{mL}{ }^{-1}$ (palmitoleic acid for $S$. aureus), 6.25 1600 $\mu \mathrm{g} \mathrm{mL}^{-1}$ (palmitoleic acid for $S$. epidermidis), 62.5 $16000 \mu \mathrm{g} \mathrm{m}^{-1}$ (lactic acid for $S$. aureus), and 50.0 $12800 \mu \mathrm{g} \mathrm{mL} \mathrm{m}^{-1}$ (lactic acid for $S$. epidermidis).

To evaluate the combined effect, $20 \mu \mathrm{L}$ of myristic acid ethanol solution, $20 \mu \mathrm{L}$ of ethanol solution of the additive, 
$1000 \mu \mathrm{L}$ of liquid medium containing beef extract $(0.01 \mathrm{~g})$, polypeptone $(0.02 \mathrm{~g})$, and sodium chloride $(0.01 \mathrm{~g}), 940 \mu \mathrm{L}$ of phosphate buffer solution, and $20 \mu \mathrm{L}$ of a bacterial dispersion $\left(3 \times 10^{5} \sim 2 \times 10^{6} \mathrm{CFU} \mathrm{mL}^{-1}\right)$ were mixed in a test tube. When lactic acid was added, $1000 \mu \mathrm{L}$ of the liquid medium, $940 \mu \mathrm{L}$ of phosphate buffer solution containing lactic acid, and $20 \mu \mathrm{L}$ of bacterial solution were added to $40 \mu \mathrm{L}$ of myristic acid ethanol solution. Lactic acid was dispersed in a phosphate buffer solution at $\mathrm{pH} 6$ because the antibacterial effect of organic acid is dependent on the $\mathrm{pH}^{20)}$.

The prepared sample $(100 \mu \mathrm{L})$ was added to a 96 -well TrueLine cell culture plate. The $\mathrm{OD}_{660}$ of these samples was evaluated by grating microplate reader (SH-1200 Lab). The conditions were as follows: wavelength $=660 \mathrm{~nm}$ and number of flashes $=10$. Samples containing $S$. aureus or $S$. epidermidis were shaken at $1000 \mathrm{rpm}$ by PSU-2T and were incubated at $37^{\circ} \mathrm{C}$ for 24 and $48 \mathrm{~h}$, respectively. To confirm the reproducibility, two pairs of plates containing the same composition were prepared and were evaluated twice. In order to calculate the FIC index and 5 FIC, the obtained turbidity was shown in a $9 \times 9$ board diagram. This turbidity is the difference between the turbidities at 0 $\mathrm{h}$ and 24 (or 48) h to exclude the effect of the precipitation of components. The $\Sigma$ FIC was calculated for each antibacterial combination as the sum of the individual FICs. The turbidity of the samples at MIC used for the calculation of FIC was $<0.010$ for all combinations. Under these conditions, no turbidity was observed in visual observation. In the board diagram, the mean turbidity values are shown in five levels, $\mathrm{OD}_{660}=0.000 \sim 0.099,0.100 \sim 0.199,0.200 \sim$ $0.299,0.300 \sim 0.399$, and above 0.400 . In the case of myristic acid/lactic acid mixed system, $100 \mu \mathrm{L}$ of a bacteria medium was spread on an agar medium containing 2.3 wt\% of agar powder to confirm the growth of $S$. aureus at $37^{\circ} \mathrm{C}$. The final concentrations of myristic acid and lactic acid were $2.45 \sim 628$ and $2000 \mu \mathrm{g} \mathrm{mL}^{-1}$, respectively.

\subsection{Determination of the combination effect}

The FIC index of the mixed system consisting of drugs A and $\mathrm{B}$ was calculated using the following equation:

$$
\begin{aligned}
& \text { FIC index }= \\
& \frac{\text { MIC of tested A in combination }}{\text { MIC of tested A only }}+\frac{\text { MIC of tested B in combination }}{\text { MIC of tested B only }}
\end{aligned}
$$

Based on the $\Sigma$ FIC values, the interactions between drugs $\mathrm{A}$ and $\mathrm{B}$ were categorized into the following four effects: (1) Synergistic effect: When $\Sigma F I C \leq 0.5$, the effect of the two drugs combined was much stronger than the sum of the effects of the single drug. (2) Additive effect: When $0.5<\Sigma$ FIC $\leq 1.0$, two drugs combined were slightly more effective than a single drug. (3) Indifferent effect: When $1.0<\Sigma$ FIC $\leq 2.0$, the combined effect is neither im- proved nor inferior to that of the single agent. (4) Antagonistic effect: When $2.0<\Sigma F I C$, the effect of the combination was inferior to the action of the single agent ${ }^{21)}$.

\section{Results}

The antibacterial activity was evaluated for mixed systems containing myristic acid and three additives including lauric acid, palmitoleic acid, and lactic acid against $S$. aureus and $S$. epidermidis. Culture medium containing $S$. aureus or $S$. epidermidis was added to a mixture of

(a)

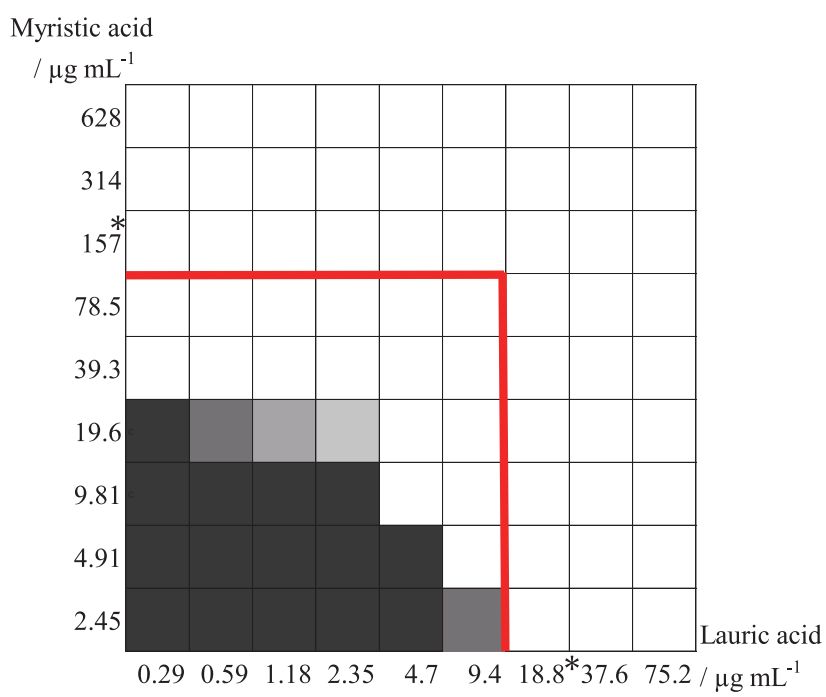

(b)

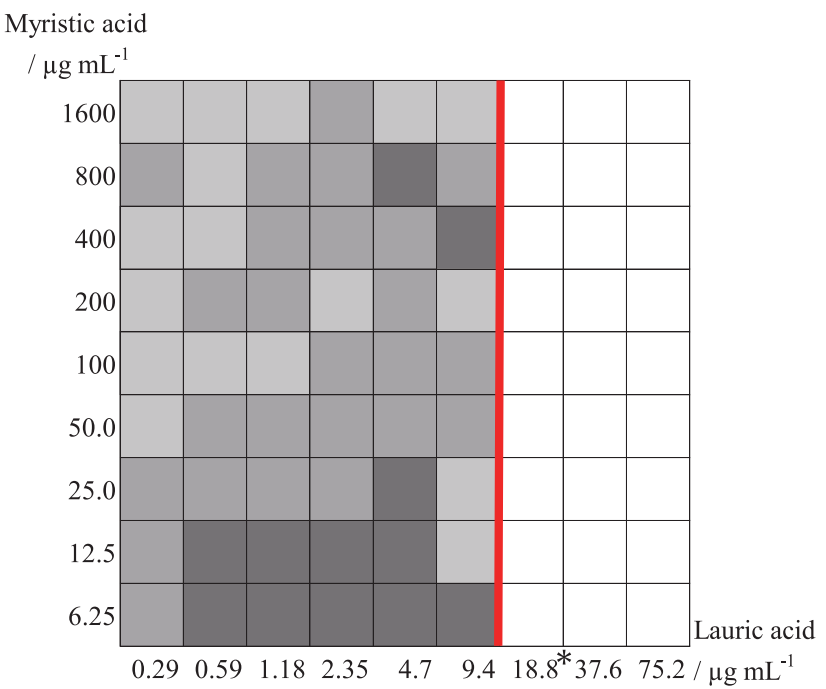

Fig. 2 Combination effects of myristic acid with lauric acid against (a) $S$. aureus (b)S. epidermidis.

๘: $\mathrm{OD}_{660}=$ above 0.400 : $\mathrm{OD}_{660}=0.300 \sim 0.399$ ㅁ: $\mathrm{OD}_{660}=0.200 \sim 0.299 \square: \mathrm{OD}_{660}=0.100 \sim 0.199 \square:$ $\mathrm{OD}_{660}=0.000 \sim 0.099 *$ : MIC of myristic acid and lauric acid, respectively. 
(a)

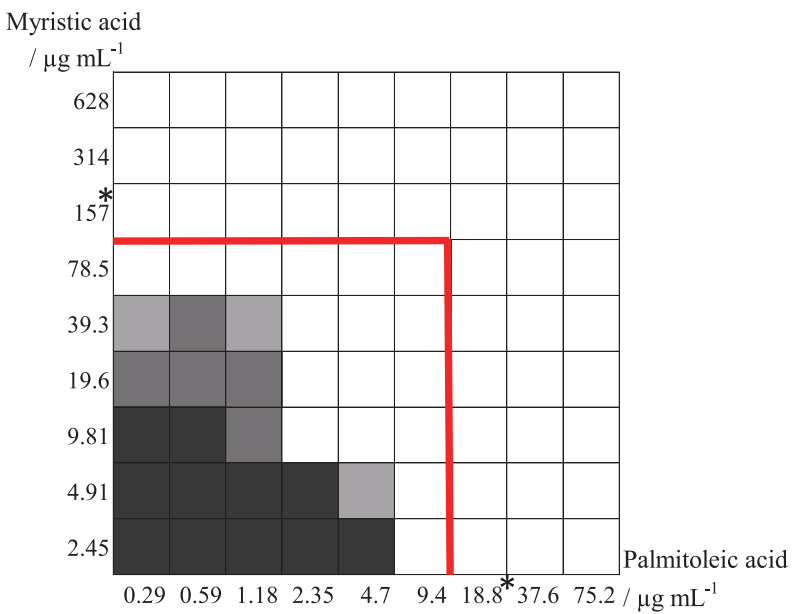

(b)

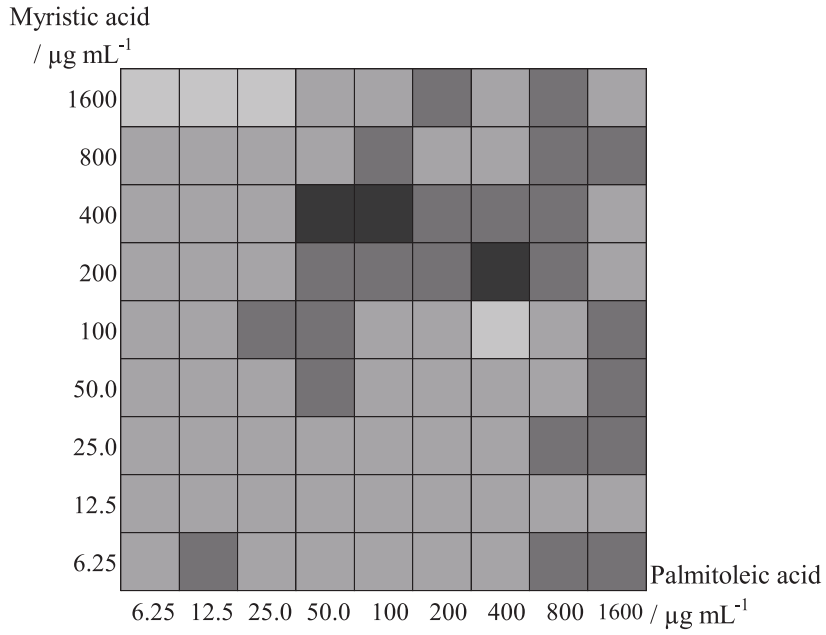

Fig. 3 Combination effects of myristic acid with palmitoleic acid against (a) $S$. aureus (b) $S$. epidermidis.

․: $\mathrm{OD}_{660}=$ above 0.400 : $\mathrm{OD}_{660}=0.300 \sim 0.399 \square$ : $\mathrm{OD}_{660}=0.200 \sim 0.299 \square: \mathrm{OD}_{660}=0.100 \sim 0.199 \square:$ $\mathrm{OD}_{660}=0.000 \sim 0.099 *$ : MIC of myristic acid and lauric acid, respectively.

myristic acid and each additives. The $\mathrm{OD}_{660}$ was measured after incubation at $37^{\circ} \mathrm{C}$ for 24 or $48 \mathrm{~h}$ for $S$. aureus and $S$. epidermidis, respectively(Figs. 2-4 and Figs. S1-S4 in supporting information).

(a) Myristic acid/lauric acid mixed system. Board diagrams of myristic acid/lauric acid mixed systems against $S$. aureus and S. epidermidis are shown in Figs. $2(\mathrm{a})$ and 2 (b), respectively. The myristic acid/lauric acid mixture exhibited an antibacterial ability against $S$. aureus as the increase of the turbidity was low $\left(\mathrm{OD}_{660}<0.010\right)$ when concentrations of myristic acid and lauric acid were above 39.3 and $18.8 \mu \mathrm{g} \mathrm{mL}{ }^{-1}$, respectively. The $\Sigma$ FIC for this mixed system was $0.56 \pm 0.12$, which suggests that the addition of (a)



(b)

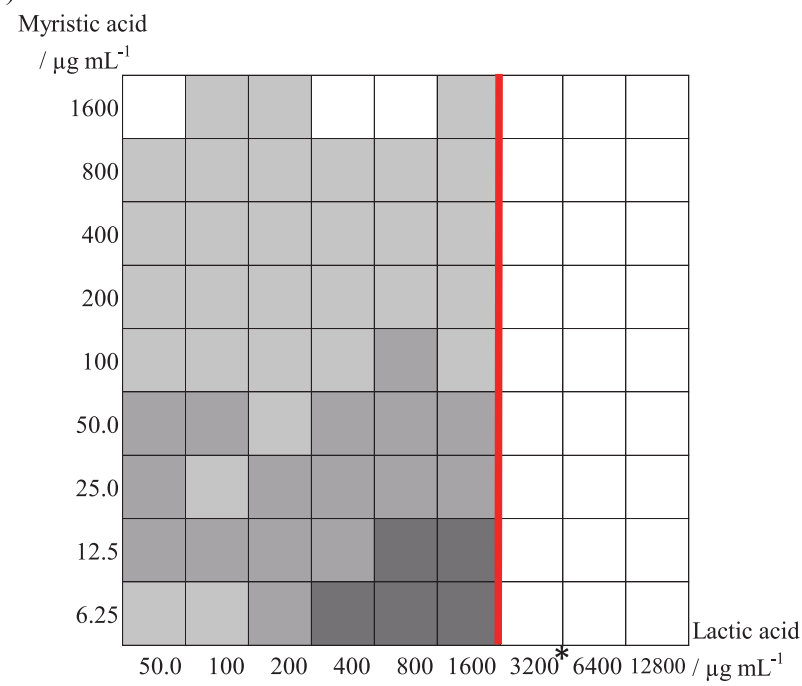

Fig. 4 Combination effects of myristic acid with lactic acid against (a)S. aureus (b)S. epidermidis.

․: $\mathrm{OD}_{660}=$ above 0.400 : $\mathrm{OD}_{660}=0.300 \sim 0.399 \square$ : $\mathrm{OD}_{660}=0.200 \sim 0.299 \square: \mathrm{OD}_{660}=0.100 \sim 0.199 \square$ : $\mathrm{OD}_{660}=0.000 \sim 0.099 *$ : MIC of myristic acid and lauric acid, respectively.

lauric acid to myristic acid induced an additive effect on the antibacterial activity against $S$. aureus (Table 2). On the other hand, turbidity did not increase against $S$. epidermidis when the concentrations of lauric acid was $\geq$ $18.8 \mu \mathrm{g} \mathrm{mL}^{-1}$ regardless of the mixing ratio of myristic acid and exhibited the antibacterial activity. This concentration was consistent with the MIC of lauric acid. This suggests that lauric acid impaired the selectivity of antibacterial activity of myristic acid.

(b) Myristic acid/palmitoleic acid mixed system. Figs. 3 
Table 2 Fractional inhibitory concentration of myristic acid in combination with lauric acid, palmitoleic acid or lactic acid against $S$. aureus or $S$. epidermidis.

\begin{tabular}{llcc}
\hline \multicolumn{1}{c}{ Strains } & \multicolumn{1}{c}{ Drug combination } & FIC index & Interpretation \\
\hline \multirow{2}{*}{ S. aureus } & Myristic acid+Lauric acid & $0.56 \pm 0.12$ & additive \\
NBRC13276 & Myristic acid+Palmitoleic acid & $0.57 \pm 0.20$ & additive \\
& Myristic acid+Lactic acid & $1.57 \pm 0.12$ & indifference \\
\hline \multirow{2}{*}{ S. epidermidis } & Myristic acid+Lauric acid & - & - \\
NBRC12993 & Myristic acid+Palmitoleic acid & - & - \\
& Myristic acid+Lactic acid & - & - \\
\hline
\end{tabular}

(a) and $\mathbf{3}$ (b) show the board diagrams of the antibacterial activity of myristic acid/palmitoleic acid mixed systems against $S$. aureus and $S$. epidermidis, respectively. The myristic acid/palmitoleic acid mixtures exhibited an antibacterial activity against $S$. aureus as the increase of the turbidity was low $\left(\mathrm{OD}_{660}<0.010\right)$ when concentrations of myristic acid and palmitoleic acid were above 78.5 and 9.4 $\mu \mathrm{g} \mathrm{mL}^{-1}$, respectively. The MICs of myristic acid and palmitoleic acid are 157 and $18.8 \mu \mathrm{g} \mathrm{mL}^{-1}$, respectively. When the two-components were mixed, a smaller amount of the components showed antibacterial activity against $S$. aureus. These results suggest that the addition of palmitoleic acid to myristic acid results in additive effect on the antibacterial behavior against $S$. aureus as $\Sigma$ FIC was 0.57 \pm 0.20 (Table 2). This mixed system did not show significant antibacterial activity against $S$. epidermidis. As shown in Fig. 3(b), the turbidity of the liquid medium increased in all compositions. Not only did neither myristic acid nor palmitoleic acid exhibit antimicrobial activity, but also no antimicrobial activity was observed under conditions where the two components were mixed.

(c) Myristic acid/lactic acid mixed system. Figs. 4(a) and 4 (b) show the board diagrams of the antibacterial activity of myristic acid/lactic acid mixed systems against $S$. aureus and $S$. epidermidis, respectively. The myristic acid/lactic acid mixture exhibited an antibacterial activity against $S$. aureus as the increase of the turbidity was low $\left(\mathrm{OD}_{660}<0.010\right)$ when the concentrations of myristic acid and lactic acid were above 78.5 and $4000 \mu \mathrm{g} \mathrm{mL}{ }^{-1}$, respectively. However, the turbidity increased specifically under all myristic acid concentrations when $2000 \mu \mathrm{g} \mathrm{mL}{ }^{-1}$ of lactic acid was added. We evaluated the system on eight plates to check for reproducibility and obtained the same results. Here, the liquid medium in which the turbidity specifically increased was spread on agar plates and incubated at $37^{\circ} \mathrm{C}$ for $24 \mathrm{~h}$ to confirm the growth of bacteria. When the liquid medium containing myristic acid and 2000 $\mu \mathrm{g} \mathrm{mL}^{-1}$ lactic acid was spread, $S$. aureus grew under all conditions: The increases in turbidity are caused by an increase in bacteria. The $\Sigma$ FIC was $1.57 \pm 0.12$, which suggested that the antibacterial activities of myristic acid and lactic acid against $S$. aureus were indifferent(Table 2). On the other hand, the antibacterial activity against $S$. epidermidis was similar to that of myristic acid/lauric acid mixed system. The myristic acid/lactic acid mixture exhibited the antibacterial activity against $S$. epidermidis when lactic acid was added above $3200 \mu \mathrm{g} \mathrm{mL}^{-1}$ regardless of the mixing ratio of myristic acid. This concentration was consistent with the MIC of lactic acid individually. When the concentrations of myristic acid and lactic acid added were 1600 and $800 \mu \mathrm{g} \mathrm{mL}{ }^{-1}$, respectively, the change in turbidity at $48 \mathrm{~h}$ was as small as 0.025 . This is because insoluble myristic acid dispersed in the medium and was the turbidity increased at $0 \mathrm{~h}$. These results suggest that lactic acid impaired the selectivity of antibacterial activity of myristic acid.

\section{Discussion}

In the mixed systems containing myristic acid, some ingredients showed additive or indifferent effects on the antimicrobial effect against $S$. aureus (Table 2). On the other hand, some of these mixed systems did not show antibacterial activity against $S$. epidermis. These differences in behavior reflect the antibacterial mechanism of each component.

First, we discuss the antibacterial behavior against $S$. aureus. The antibacterial properties against $S$. aureus were enhanced in the myristic acid/lauric acid and myristic acid/palmitoleic acid mixed systems. This effect suggests that the mixture of the two types fatty acids potentiate their action on the cell membrane. Desbois et al. reported that fatty acids affect the membrane function through the solubilization of cell membranes, disruption of electron transport chains, inhibition of oxidative phosphorylation, impaired enzyme activity, and formation of autoxidative degradation products ${ }^{22-24}$. These two ingredients act on cell membranes by the same mechanism and thus have an additive antibacterial effect.

On the other hand, synergistic antibacterial effect was not observed in the myristic acid/lactic acid mixed system. 


\section{Okukawa, Y. Yoshizaki, S. Yano et al.}

In particular, the increase of the turbidity was low when the concentration of lactic acid was above $4000 \mu \mathrm{g} \mathrm{mL}{ }^{-1}$, which is the MIC against $S$. aureus. We speculate that this result is due to the fact that the antibacterial mechanism of lactic acid is different from that of fatty acids. Undissociated acid molecules can penetrate the cell membrane and dissociate in the intracellular region, causing a $\mathrm{pH}$ decrease in the bacterial cell ${ }^{25-27)}$. The $\mathrm{pH}$ was $3.3 \sim 4.4$ for myristic acid/lactic acid mixed system when the concentration of lactic acid was above $4000 \mu \mathrm{g} \mathrm{mL}{ }^{-1}$. The $\mathrm{pH}$ of these mixed systems was out of the buffer region of the phosphate buffer ( $\mathrm{pH} 6)$, because they contained high concentration of lactic acid. Since the pKa value of lactic acid is 3.83, many lactic acid molecules exist in an undissociated state in a system under these conditions ${ }^{28)}$.

Furthermore, the turbidity specifically increased for all myristic acid concentrations when $2000 \mu \mathrm{g} \mathrm{mL} \mathrm{m}^{-1}$ of lactic acid was added(Fig. 4(a)). When these samples were spread on agar medium, the growth of $S$. aureus was confirmed. The exact mechanism of this phenomenon is not clear now. Here, we suggest a possible mechanism: Significant antibacterial effect was not observed, because the bacteria may have altered metabolic pathways and gene expression to maintain homeostasis when they were exposed to lactic acid ${ }^{29-31)}$. Rode et al. reported that in the environment acidified by lactic acid, $S$. aureus produced larger amounts of ammonium, 2,3-butanedione, and pyrazine than in the environment exposed to acetic acid or hydrochloric acid. Furthermore, the expression of genes involved in energy production was enhanced ${ }^{31)}$.

Finally, the antibacterial behavior against $S$. epidermidis is discussed. The myristic acid/lauric acid and myristic acid/lactic acid mixed systems confirmed the antibacterial activity as the increase of the turbidity was low when the concentration of lauric acid and lactic acid were 18.8 and $3200 \mu \mathrm{g} \mathrm{mL}^{-1}$, respectively, which were the MICs against $S$. epidermidis. We found that myristic acid does not show antibacterial activity against $S$. epidermidis ${ }^{10)}$. Therefore, these results suggest that myristic acid did not involve the antibacterial activity, while lauric acid and lactic acid showed antibacterial activity against $S$. epidermidis independently: Lauric acid and lactic acid inhibited the selective antibacterial properties of myristic acid against $S$. aureus.

In the case of myristic acid/palmitoleic acid mixed system, the turbidity increased under all mixed conditions: The antibacterial behavior was not observed against $S$. epidermidis. Palmitoleic acid also does not exhibit antibacterial activity against $S$. epidermidis as a single component as well as myristic acid ${ }^{10)}$. This result suggests that the selective antibacterial activity against $S$. aureus is maintained even when the two components, which do not exhibit antibacterial activity against $S$. epidermidis, are mixed. The exact mechanism of the selective antibacterial behavior is unclear. We introduce a hypothesis here. It may be influenced by differences in the composition of lipid fatty acids in the cell membranes of $S$. aureus and $S$. epidermidis. Tiwai et al. reported that the composition of branched fatty acids in the cell membrane of $S$. epidermidis is higher than that of $S$. aureus and fluidity of the cell membrane in $S$. epidermidis is higher than that in $S$. epidermidis $^{32)}$. Branched fatty acids can disrupt the order of cell membranes more than straight-chain fatty acids and increase membrane permeability and fluidity of the cell membrane $^{33)}$. Furthermore, the cell membrane of $S$. aureus contains staphyloxanthin, which is a carotenoid that contributes to membrane stabilization, while this component is absent in S. epidermidis ${ }^{34,35)}$.

\section{Conclusion}

The antibacterial activity of the two-component mixed system of lauric acid, palmitoleic acid, and lactic acid with myristic acid was evaluated against $S$. aureus and $S$. epidermidis using the checkerboard method. The antibacterial activity against $S$. aureus was enhanced in the myristic acid/lauric acid and myristic acid/palmitoleic acid mixed systems. The myristic acid/lactic acid mixed system did not significant change in the antibacterial properties of each substances. On the other hand, myristic acid/palmitoleic acid mixed system did not show clear antibacterial activity against $S$. epidermidis. However, myristic acid/lauric acid mixed system and myristic acid/lactic acid mixed system exhibited the antibacterial behavior against $S$. epidermidis when the concentration of lauric acid and lactic acid above MIC, respectively. These results suggest that the property of myristic acid, which shows the selective antibacterial activity against $S$. aureus as a single component, may be altered depending on the coexisting components. Antibacterial activity of mixed fatty acids must be related to the physical properties of mixed fatty acids. It has been reported that a decrease in Kraft point, pKa, and water evaporation through molecular membrane occurs in mixed systems containing two types of fatty acids ${ }^{36-38)}$. These physical phenomena are caused by the packing change, which increases the intermolecular distance. In the future, systematic analysis between molecular structure, interfacial chemistry, and biological properties will lead to a better understanding of the behavior of molecules on biological surfaces. These findings of this study are useful for cosmetic and body cleansers formulation design containing fatty acids.

\section{Acknowledgment}

This study was partly supported by the Yamagata Uni- 
versity YU-COE (C) program.

\section{Supporting Information}

This material is available free of charge via the Internet at doi: $10.5650 /$ jos.ess21090

\section{References}

1) Kanicky, J.R.; Poniatowski, A.F.; Mehta, N.R.; Shah, D.O. Cooperativity among molecules at interfaces in relation to various technological processes: effect of chain length on the pKa of fatty acid salt solutions. Langmuir 16, 172-177 (2000).

2) Becker, L.C.; Bergfeld, W.F.; Belsito, D.V.; Hill, L.A.; Klaassen, C.D.; Marks Jr, J.D.; Shank, R.C.; Slaga, T.J.; Snyder, P.W.; Andersen, F.A. Final report of the amended safety assessment of myristic acid and its salts and esters as used in cosmetics. Int. J. Toxicol. 29, 162S-186S (2010).

3) Nakatsuji, T.; Kao, M.C.; Fang, J.Y.; Zouboulis, C.C.; Zhang, L.; Gallo, R.L.; Huang, C.M. Antimicrobial property of lauric acid against Propionibacterium acnes: its therapeutic potential for inflammatory acne vulgaris. J. Invest. Dermatol. 129, 2480-2488(2009).

4) Chao-Hsuan, C.; Wang, Y.; Nakatsuji, T.; Liu, Y.T.; Zouboulis, C.C.; Gallo, R.L.; Zhang, L.; Hsieh, M.F.; Huang, C.M. An innate bactericidal oleic acid effective against skin infection of methicillin-resistant Staphylococcus aureus: A therapy concordant with evolutionary medicine. J. Microbiol. Biotechnol. 21, 391399 (2011).

5) Moran, J.C.; Alorabi, J.A.; Horsburgh, M.J. Comparative transcriptomics reveals discrete survival responses of $S$. aureus and $S$. epidermidis to sapienic acid. Front. Microbiol. 8, 1-12 (2017).

6) Yamamoto, Y.; Kawamura, Y.; Yamazaki, Y.; Kijima, T.; Morikawa, T.; Nonomura, Y. Palmitoleic acid calcium salt: A lubricant and bactericidal powder from natural lipids. J. Oleo Sci. 64, 283-288(2015).

7) Yamamoto, Y.; Kijima, T.; Morikawa, T.; Nonomura, Y. Lubricant and bactericidal properties of calcium salts of fatty acids: Effect of degree of unsaturation. J. Oleo Sci. 64, 1095-1100 (2015).

8) Yamamoto, Y.; Morikawa, T.; Kawai, T.; Nonomura, Y. Selective bactericidal activity of divalent metal salts of lauric acid. ACS Omega 2, 113-121 (2017).

9) Morikawa, T.; Yamamoto, Y.; Nonomura, Y. Effect of $\mathrm{pH}$ on bactericidal activities of calcium laurate. J. Oleo Sci. 67, 859-862 (2018).

10) Watanabe, T.; Yamamoto, Y.; Miura, M.; Konno, H.; Yano, S.; Nonomura, Y. Systematic analysis of selective bactericidal activity of fatty acids against Staphylococcus aureus with minimum inhibitory concentration and minimum bactericidal concentration. J. Oleo Sci. 68, 291-296 (2019).

11) Levine, A.S.; Fellers, C.R. Action of acetic acid on food spoilage microorganisms. J. Bacteriol. 39, 499-515 (1940).

12) Goepfert, J.M.; Hicks, R. Effect of volatile fatty acids on Salmonella typhimurium. J. Bacteriol. 97, 956958 (1969).

13) Yamamoto, Y.; Higashi, K.; Yoshii, H. Inhibitory activity of organic acids on food spoilage bacteria. Nippon Shokuhin Kogyo Gakkaishi 31, 525-530 (1984).

14） Matsuda, T.; Yano, T.; Maruyama, A.; Kumagai, H. Antibacterial activities of organic acids determined by minimum inhibitory concentrations at different $\mathrm{pH}$ ranged from 4.0 to 7.0. Nippon Shokuhin Kogyo Gakkaishi 41, 687-702(1994).

15) Berenbaum, M.C. A method for testing for synergy with any number of agents. J. Infect. Dis. 137, 122130 (1978).

16) Hsieh, M.H.; Yu, C.M.; Yu, V.L.; Chow, J.W. Synergy assessed by checkerboard a critical analysis. Diagn. Microbiol. Infect. Dis. 16, 343-349 (1993).

17) Okukawa, M.; Yoshizaki, Y.; Mayu, T.; Yano, S.; Nonomura, Y. Antibacterial activity of the mixed systems containing 1,2-dodecanedo against Staphylococcus aureus and Staphylococcus epidermidis. J. Oleo Sci. in press.

18) Rawling, A.V.; Davies, A.; Carlomusto, M.; Pillai, S.; Zhang, K.; Kosturko, R.; Verdejo, P.; Feinberg, C.; Nguyen, L.; Chandar, P. Effect of lactic acid isomers on keratinocyte ceramide synthesis, stratum corneum lipid levels and stratum corneum barrier function. Arch. Dermatol. Res. 288, 383-390(1996).

19) Proksch, E. pH in nature, humans and skin. J. Dermatol. 45, 1044-1052 (2018).

20) Chaveerach, P.; Keuzenkamp, D.A.; Urlings, H.A.P.; Lipman, L.J.A.; van Knapen, F. In vitro study on the effect of organic acids on Campylobacter jejuni/coli populations in mixtures of water and feed. Poult. Sci. 81, 621-628(2002).

21) Chaveerach, P.; Keuzenkamp, D.A.; Urlings, H.A.P.; Lipman, L.J.A.; van Knapen, F. In vitro study on the effect of organic acids on Campylobacter jejuni/coli populations in mixtures of water and feed. Poult. Sci. 81, 621-628(2002).

22) Zheng, C.J.; Yoo, J.S.; Lee, T.G.; Cho, H.Y.; Kim, Y.H.; Kim, W.G. Fatty acid synthesis is a target for antibacterial activity of unsaturated fatty acids. FEBS Lett. 579, 5157-5162 (2005).

23) Desbios, A.P.; Smith, V.J. Antibacterial free fatty acids: activities, mechanism of action and biotechnological potential. Appl. Microbiol. Biothecnol. 85, 1629-1642 
(2010).

24) Fischer, C.L.; Darke, D.R.; Dawson, D.V.; Blanchette, D.R.; Brogden, K.A.; Wertz, P.W. Antibacterial activity of sphingaoid bases and fatty acids against gram-positive and gram-negative bacteria. Antimicrob. Agents Chemother. 56, 1157-1161 (2012).

25) Cherrington, C.A.; Hinton, M.; Mead, G.C.; Chopra, I. Organic acids: Chemistry, antibacterial activity and practical applications. Adv. Microb. Physiol. 32, 87108 (1991).

26) Alakomi, H.-L.; Skytta, E.; Saarela, M.; Mattila, T.; Mattila-sandholm, T.; Latva-kala, K.; Helander, I.M. Lactic acid pemeabilizes gram-negative bacteria by disrupting the outer membrane. Appl. Environ. Microbiol. 66, 2001-2005 (2000).

27) Ricke, S.C. Perspectives on the use of organic acids and short chain fatty acids as antimicrobials. Poult. Sci. 82, 632-639(2003).

28) Dibner, J.J.; Buttin, P. Use of organic acids as a model to study the impact of gut microflora on nutrition and metabolism. J. Appl. Poult. Res. 11, 453-463 (2002).

29) Aiba, H.; Mizuno, T. Adaptive responses and transduction in bacteria. Nippon Saikingaku Zasshi 51, 549557 (1996).

30) Weinrick, B.; Dunman, P.M.; McAleese, F.; Murphy, E.; Projan, S.A.; Fang, Y.; Novick, R.P. Effect of mild acid on gene expression in Staphylococcus aureus. J. Microbiol. 186, 8407-8423(2004).

31) Rode, T.M.; Moretro, T.; Langsrud, S.; Langsrud, O.; Vogt, G.; Holck. Responses of Staphylococcus aureus exposed to $\mathrm{HCl}$ and organic acid stress. Can. J. Microbiol. 56, 777-792(2010).

32) Tiwari, K.B.; Gatto, C.; Wilkinson, B.J. Plasticity of coagulase-negative staphylococcal membrane fatty acid composition and implications for responses to antimicrobial agents. Antibiotics 9, 214-224(2020).

33) Zhang, Y.M.; Rock, C.O. Membrane lipid homeostasis in bacteria. Nat. Rev. Microbiol. 6, 222-233 (2008).

34) Chamberlain, N.R.; Mehrtens, B.G.; Xiong, Z.; Kapral, F.A.; Boardman, J.L.; Rearick, J.I. Correlation of carotenoid production, decreased membrane fluidity, and resistance to oleic acid killing in Staphylococcus aureus 18Z. Infect. Immun. 59, 4332-4337(1991).

35) Mishra, N.N.; Liu, G.Y.; Yeaman, M.R.; Nast, C.C.; Proctor, R.A.; McKinnell, J.; Bayer, A.S. Carotenoid-related alteration of cell membrane fluidity impacts Staphylococcus aureus susceptibility to host defense peptides. Antimicrob. Agents Chemother. 55, 526-531 (2011).

36) Ogino, K.; Okamoto, M. Study on the melting behavior of odd-even fatty acids mixtures. J. Jpn. Oil Chemists Soc. 24, 474-477(1975).

37) Shiao, S.Y.; Patist, A.; Free, M.L.; Chhabra, V.; Huibers, P.D.T.; Gregory, A.; Patel, S.; Shah, D.O. The importance of sub-angstrom distances in mixed surfactant systems for technological processes. Colloids Surf. A 128, 197-208 (1997).

38) Kanicky, J.R.; Shah, D.O. Effect of premicellar aggregation on the pKa of fatty acid soap solutions. Langmuir 19, 2034-2038(2003).

CC BY-SA 4.0 (Attribution-ShareAlike 4.0 International). This license allows users to share and adapt an article, even commercially, as long as appropriate credit is given and the distribution of derivative works is under the same license as the original. That is, this license lets others copy, distribute, modify and reproduce the Article, provided the original source and Authors are credited under the same license as the original. 